\title{
TAGUNG
}

\section{Deutschlands Rolle in Europa: Führungsmacht oder Vetospieler?}

\author{
Moritz Eckert und Insa Nieberg*
}

Europa ist zweifelsfrei in Bewegung: Die Finanzkrise, die Revolutionen in Nordafrika und die Möglichkeit neuer EU-Erweiterungen beeinflussen das Umfeld, in dem die Europäische Union agiert, beschränken angestammte Handlungsspielräume und schaffen andernorts neue Perspektiven. Die Jahreskonferenz des Instituts für Europäische Politik e.V. (IEP) in Zusammenarbeit mit dem Wissenschaftlichen Direktorium trug diesen Entwicklungen Rechnung und analysierte deren Implikationen aus nationaler, europäischer und internationaler Perspektive. Sowohl die sich verschärfende Staatsschuldenkrise als auch die Umstürze in Nordafrika waren Entwicklungen, die wegen ihrer Geschwindigkeit rasche Reaktionen vonseiten der Europäischen Union und ihrer Mitgliedstaaten erforderten. Aufgrund des großen Drucks und in Ermangelung eines Präzedenzfalles für derartige Herausforderungen wichen einige Akteure, insbesondere auch die Bundesrepublik, von klassischen Handlungsmustern ab. Dies wiederum schuf neue Präzedenzfälle und stellte das Krisenmanagement vor zusätzliche Herausforderungen.

Dreh- und Angelpunkt aller Debatten war somit die Frage, wie Deutschland sich angesichts grundlegend veränderter Ausgangsbedingungen als Akteur innerhalb der Europäischen Union und darüber hinaus positioniert. Denn während Deutschland trotz Finanz- und Wirtschaftskrise von allen EU-Mitgliedstaaten wirtschaftlich noch am besten dasteht, wurde der Großteil des Währungsraums von einer starken Rezession erfasst.

\section{„Deutschland in der Europäischen Union: Führungsmacht oder Vetospieler?“"}

Jahrestagung des Instituts für Europäische Politik (IEP) in Zusammenarbeit mit dem Wissenschaftlichen Direktorium

Mit freundlicher Unterstützung der Fritz Thyssen Stiftung

Berlin, 6.-7. Oktober 2011

\section{Begrïßung}

Jürgen LENNARTZ, MDg, Bevollmächtigter des Saarlandes beim Bund

Prof. Dr. Michael KREILE, Vorsitzender des Wissenschaftlichen Direktoriums; HumboldtUniversität zu Berlin

Prof. Dr. Mathias JOPP, Direktor, Institut für Europäische Politik (IEP), Berlin

Eine neue deutsche Europapolitik - Führungsmacht oder Vetospieler?

Vorsitz

Prof. Dr. Heinrich SCHNEIDER, Ehrenvorsitzender des Wissenschaftlichen Direktoriums; Universität Wien

Kurzstatements

VLR I Dr. Peter SCHOOF, Beauftragter für Grundsatzfragen der EU-Außenbeziehungen sowie der Beziehungen zu den Mitgliedstaaten der EU, Auswärtiges Amt, Berlin

Prof. Dr. Gisela MÜLLER-BRANDECK-BOCQUET, Universität Würzburg

Prof. Dr. Werner WEIDENFELD, Universität München

Prof. Dr. Wolfgang WESSELS, Universität zu Köln

* Moritz Eckert, Institut für Europäische Politik e.V., Berlin. Insa Nieberg, Institut für Europäische Politik e.V., Berlin. 
Die relative wirtschaftliche Stärke macht deutsche Beteiligungen an Rettungsmaßnahmen für ,Defizit-Sünder ' im eigenen Land immer unpopulärer. Gleichzeitig ist in den europäischen Nachbarstaaten eine wachsende Frustration über die als zögerlich und bisweilen sprunghaft empfundene deutsche Haltung spürbar. In diesem Sinne wurde auch grundsätzlich erörtert, warum und in welchem Ausmaß die Mitgliedstaaten dieses Europa angesichts steigender Kosten zu tragen bereit sind und welche Perspektiven für eine Harmonisierung der nationalen Politiken bestehen.

Deutschlands Positionierung in und zu Europa

Das Auftaktpanel ging der Frage nach, wie die Bundesregierung auf die Veränderungen im europäischen und globalen Umfeld reagiert. Einigkeit herrschte darüber, dass die Rahmenbedingungen wesentlich für eine $\mathrm{Bi}$ lanz der deutschen Politik seien. Die Frage nach der Rolle Deutschlands sei auch bestimmt durch externe Faktoren. So hätte die Finanzkrise die Europäische Union unvorbereitet und inmitten einer Konsolidierungsphase getroffen. Die Überforderung des europäischen Krisenmanagements angesichts dieser Umwälzungen könnte als erster Bruch des Bildes von Frieden und Stabilität, das die Basis der Europäischen Union ist, bezeichnet werden. Darüber hinaus befinde sich die Europäische Union in Bezug zu ihren Bürgerinnen und Bürgern in einer Legitimationskrise. Deutlich wurde, dass Deutschland in diesem Prozess eine vergleichsweise schwierige Position einnimmt: Während einerseits erwartet werde, dass die Bundesrepublik handle, so werde andererseits eine ,deutsche Dominanz ebenso kritisiert.

Als prägend für die deutsche Politik wurde das Novum der gegenwärtigen Situation betont: Mit Blick auf den Militäreinsatz in Libyen und die Debatte über Eurobonds zeige sich, dass das ,traditionelle Duo', Deutschland und Frankreich, bisweilen getrennte Wege gehe. Gerade die Finanzkrise mache
Deutschland als Gläubiger und Zahlmeister: Die Stabilisierung der Eurozone und die Haushaltsplanung 2014-2020

Vorsitz

Prof. Dr. Rolf CAESAR, Universität Hohenheim, Stuttgart

Kurzstatements

Prof. Dr. Ansgar BELKE, Universität DuisburgEssen; Deutsches Institut für Wirtschaftsforschung (DIW), Berlin

Dr. Friedrich HEINEMANN, Zentrum für Europäische Wirtschaftsforschung (ZEW), Mannheim Prof. Dr. Peter-Christian MÜLLER-GRAFF, Universität Heidelberg

VLR Dr. Peter PTASSEK, Referatsleiter E04 Wirtschafts- und Währungsunion, Auswärtiges Amt, Berlin

Die GASP/GSVP zwischen Vertragstext, ,coalitions of the willing" und nationalen Alleingängen Vorsitz

Prof. Dr. Hanspeter NEUHOLD, Universität Wien Einführung

Dr. Hans-Dieter LUCAS, MDg, Politischer Direktor, Referat EU-KOR, Auswärtiges Amt, Berlin

Kurzstatements

Prof. Dr. Mathias JOPP, Direktor, Institut für Europäische Politik (IEP), Berlin

Dr. Ronja KEMPIN, Leiterin der Forschungsgruppe EU-Außenbeziehungen, Stiftung Wissenschaft und Politik, Berlin

\section{Dinner Speech}

Peter ALTMAIER, MdB, Parl. Staatssekretär a.D., Erster Parlamentarischer Geschäftsführer der CDU/CSU-Fraktion des Deutschen Bundestages, Berlin

Die neuen Rollen Deutschlands aus der Sicht europäischer Partnerstaaten

Vorsitz

Prof. Dr. Joachim SCHILD, Universität Trier

Kurzstatements

Prof. Dr. William E. PATERSON, Aston University, Birmingham

Prof. Dr. Anne-Marie LE GLOANNEC, Forschungsdirektorin, Centre d'études et de recherches internationales (CERI), Paris

Dr. Petr DRULÁK, Direktor, Institute of International Relations, Prag

Prof. Angelo BOLAFFI, Universität La Sapienza, Rom 
den Zwiespalt deutscher Politik deutlich: Einerseits sei Deutschland Führungsmacht und Gestalter der EU-Prozesse, andererseits übernehme die Bundesregierung an anderen Stellen auch eine bremsende Rolle, etwa bei den Diskussionen um Eurobonds und die angestrebte Rolle der Europäischen Zentralbank (EZB). Die gegenwärtige Situation könnte für die Europäische Union einen Scheideweg darstellen, an dem kleinere Gruppen von Mitgliedstaaten sich für einen eigenen Pfad tieferer Integration entschieden. Dies gelte, auch wenn in Bezug auf die konkrete Ausgestaltung zwischen den einzelnen Politikfeldern zu differenzieren sei, sowohl für die Gemeinsame Außen- und Sicherheitspolitik (GASP) als auch die Wirtschafts- und Währungsunion (WWU). Lösungsvorschläge hätten jedoch in beiden Fällen vor allem die großen Mitgliedstaaten im Blick. Eine kollektive ,leadership"Struktur hingegen würde gewährleisten, dass alle Mitgliedstaaten eingebunden sind, und so ein Europa der zwei Geschwindigkeiten verhindern.

Wie schon die Diskussionen um die WWU und die GASP zeigten, basierten die Diskurse über die EU-Politik vor allem auf der (medialen) Wahrnehmung dieser. Die Deutung der konkreten Ausgestaltung einzelner Politikfelder hänge eng mit der Legitimationsbasis der Europäischen Union zusammen. Die Bürgerinnen und Bürger erwarteten mehr Transparenz angesichts des steigenden Brüsseler Einflusses auf viele Lebensbereiche. So sei gegenwärtig eine neue, grundlegende Strategie vonnöten, die offen kommuniziert werden müsse. Dabei seien Kosten und Nutzen der Entscheidungen abzuwägen und den Bürgern, anders als in der Vergangenheit, umfassend $\mathrm{zu}$ vermitteln. Während bei bisherigen Vertragsänderungen und der Aufnahme neuer Mitgliedstaaten politische Argumente oftmals schwerer gewogen hätten als wirtschaftliche, stelle sich nun die Frage nach der Legitimation einer fortschreitenden Integration im wirtschaftlichen Bereich.
Innerstaatliche Vetospieler in der deutschen Europapolitik: Bundesverfassungsgericht und Bundestag

Vorsitz

Prof. Dr. Franz MAYER, Universität Bielefeld

Kurzstatements

Prof. Dr. Ingolf PERNICE, Humboldt-Universität zu Berlin

Prof. Dr. Christine LANDFRIED, Universität Hamburg

Dr. Sven VOLLRATH, Leiter Referat Europa, Deutscher Bundestag, Berlin

\section{Schlussfolgerungen}

Prof. Dr. Michael KREILE, Vorsitzender des Wissenschaftlichen Direktoriums; HumboldtUniversität zu Berlin

Prof. Dr. Mathias JOPP, Direktor, Institut für Europäische Politik (IEP), Berlin

Nichtsdestotrotz sei es eine Leitlinie der deutschen Europapolitik, den bisherigen Stand der europäischen Integration zu wahren. Die Frage bleibe aber, wie danach weiter zu verfahren sei. Zwar müsse einer Zersplitterung der Europäischen Union vorgebeugt werden, dennoch gebe es die Option intergouvernementaler Initiativen kleinerer Gruppen in Bereichen, die nicht gemeinschaftlich geregelt seien. Die deutsche Politik stehe somit vor der Aufgabe, sich in und zu Europa zu positionieren. Dabei werde die Zukunft zeigen, ob Deutschland innerhalb des Integrationsprozesses als Gestalter oder Bremser, als Teil einer Gruppe besonders integrationswilliger Staaten, als (Mit-)Führungsmacht oder Hegemon wider Willen agieren werde.

\section{EU-Haushalt in Zeiten der Krise: Finanzie- rungsperspektiven jenseits der deutschen Ge- berrolle}

Im zweiten Panel stand die kontinuierlich ungleiche finanzielle Belastung der EU-Mitgliedstaaten im Vordergrund. Die Tatsache, dass sowohl Rettungsschirme als auch die Nettozahlungen des EU-Haushalts stets von den gleichen Staaten getragen würden, führe insbesondere in Krisenzeiten immer wieder 
zu hitzigen und oft wenig sachlichen öffentlichen Debatten. Insbesondere in Deutschland, das Gläubiger der Krisenstaaten und oft zitierter ,Zahlmeister" der Europäischen Union sei, werde in der Krise von Medien und Bürgern der ökonomische Mehrwert der fortgesetzten Mitgliedschaft wiederholt infrage gestellt.

Auch aus dem Wunsch heraus, die Staaten finanziell zu entlasten und den EU-Haushalt auf eine breitere Grundlage zu stellen, seien in letzter Zeit insbesondere die Vorschläge einer Finanztransaktionssteuer oder EU-Mehrwertsteuer diskutiert worden. Doch während damit in der Theorie neue Einnahmequellen erschlossen würden und dies möglicherweise den Anstoß zu einer intensiveren Auseinandersetzung mit der Europäischen Union liefern könnte, wurden beide Ideen mehrheitlich ablehnend bewertet. Angesichts der Tatsache, dass eine Finanztransaktionssteuer den Normalbürger kaum beträfe und eine EUMehrwertsteuer zu einer weiteren Umverteilung zu Lasten der Geringverdiener führe, sei das heutige System deutlich geeigneter. Allerdings habe sich die EU-Struktur- und Kohäsionspolitik in der Vergangenheit nicht als erfolgreich erwiesen. Um die Wirkung dieser Politiken zu verbessern, könnten künftig auch Reformen wie zum Beispiel eine effizientere Verwaltung mit EU-Mitteln gefördert werden. Um dabei Mehrkosten zu vermeiden, könnte auf eine höhere nationale Kofinanzierung der Programme oder einen allgemeinen Korrekturmechanismus mit bestimmten Höchstgrenzen für den Nettofluss der Gelder gedrängt werden. Es gelte, die vorhandenen Gelder besser auszugeben, um die Wettbewerbsfähigkeit zu steigern und Wachstumsimpulse zu setzen, anstatt immer mehr auszugeben.

Insgesamt ließe sich europaweit beobachten, dass die Grenzen der Solidarität allmählich erreicht würden. So hätten etwa die Slowakei und die Niederlande der Ausweitung der European Financial Stability Facility (EFSF) nur zögerlich zugestimmt, während die Debatte in Deutschland vom Bild des ,Zahlmeisters' Europas dominiert würde. Die Tatsache, dass bisherige Rettungspakete wenig erfolgreich gewesen seien, in den Peripherie-Staaten die Zinsen auf Staatsanleihen kontinuierlich stiegen und es in der Krisenbekämpfung insgesamt an Kohärenz fehle, habe dem Euroskeptizismus hierzulande Auftrieb gegeben. Durch die Gläubigerschaft einerseits und den größten Anteil am EU-Haushalt andererseits habe Deutschland in der Krise auf beiden Schultern am stärksten zu tragen. Insbesondere die komplexe Verflechtung der verschiedenen Krisenbekämpfungsmaßnahmen - angefangen von den Griechenland-Paketen über die Stabilisierungsmechanismen bis hin zum am besten geeigneten Einsatz der EZB - biete ein für Politiker, Medien und Bevölkerung kaum zu verstehendes Gesamtbild. Das tatsächliche Ausmaß deutscher Zahlungsverpflichtungen und Risiken könne dabei kaum valide beziffert werden. Angesichts der Tatsache, dass Deutschland der mit Abstand größte Nettozahler der Europäischen Union sei, führe dies zu einem verstärkten Hinterfragen der Sinnhaftigkeit einer fortgesetzten EU-Mitgliedschaft. Die so vermittelte Unsicherheit wirke sich wiederum direkt auf die Finanzmärkte aus.

Gleichzeitig wurde die gegenwärtige Krise vonseiten der Teilnehmer auch wiederholt als Chance für die Europäische Union gedeutet, wenn in den Verhandlungen das Krisenmanagement mit Konzepten der EU-Finanzierung verbunden werde. Dadurch könnten sowohl die Kohärenz europäischer Wirtschaftspolitik als auch die wirtschaftliche und politische Integration verstärkt werden. Dies beinhalte die Notwendigkeit einer vertieften europäischen Debatte, die bislang nicht stattgefunden habe. Als unmittelbarer Schritt des aktuellen Krisenmanagements wurde ein als essenziell angesehener Schuldenschnitt für Griechenland diskutiert, infolge dessen die EZB sich wieder verstärkt ihren ursprünglichen Aufgaben widmen könnte. Längerfristig sei ein koordiniertes Programm zum Abbau der Staatsverschuldungen der Mitgliedstaaten der Europäischen Union notwendig, das von nationalen Parlamenten beschlossen und kon- 
sequent umgesetzt werden müsse. Mit den bisherigen Instrumenten hingegen stoße man an Grenzen und habe unter dem Druck der Anleger bereits einige überschritten. So seien zum Beispiel die Aufkäufe von Staatsanleihen der Krisenstaaten durch die EZB sehr umstritten. Da diese bis zur Operationalisierung des Europäischen Stabilitätsmechanismus (ESM) jedoch die einzige europäische Institution sei, die am Markt interveniere, häuften sich zur Zeit die Versuche der politischen Einflussnahme - eine Entwicklung, die von den meisten der versammelten Experten sehr kritisch bewertet wurde.

Erfolge und Scheitern in Außen- und Sicherheitspolitik

Das dritte Panel befasste sich mit den jüngsten Entwicklungen in der gemeinsamen $\mathrm{Au}$ Ben-, Sicherheits- und Verteidigungspolitik. Im Zentrum der kontrovers geführten Debatte standen der Versuch einer Bilanz der GASP seit ,Lissabon“ und die Frage, welche Tendenzen für die Weiterentwicklung des Politikbereichs daraus abgeleitet werden können.

$\mathrm{Zu}$ Beginn der Sitzung wurden einige erste positive Ergebnisse der GASP reflektiert. So habe die Europäische Union im Nahost-Friedensprozess deutlich an Gewicht gewonnen und sich vom ,payer" zum , player" entwickelt. Ebenso seien die unter der Ägide des Europäischen Auswärtigen Dienstes stattfindenden Gespräche zwischen Kosovo und Serbien wie auch eine bessere Sichtbarkeit der Europäischen Union bei den Vereinten Nationen als Erfolge zu bewerten. Darüber hinaus habe die GASP in der Libyen-Krise im Hintergrund gut funktioniert, wie etwa die schnelle Einigung auf ein Waffenembargo, Wirtschaftssanktionen und Reiseverbote, die Delegitimierung Muammar al-Gaddafis als Vertreter des libyschen Volkes, die Anerkennung des Übergangsrates sowie die Neuausrichtung der Nachbarschaftspolitik zeigten. Dennoch sei der Gesamtprozess nicht immer reibungslos verlaufen und teilweise von Vertragsverletzungen, wie etwa Verstößen von Mitglied- staaten gegen die Konsultationspflicht oder das Prinzip der konsensualen Abstimmung in internationalen Organisationen, geprägt gewesen.

Diese oftmals charakteristische Dissonanz wurde als die größte Herausforderung einer kohärenten europäischen Außen- und Sicherheitspolitik gewertet. Nach wie vor prägten nationale Alleingänge und Profilierungsbestrebungen das Tagesgeschäft. Oftmals verhinderten dabei die kollidierenden Ambitionen gerade der einflussreichsten Staaten Deutschland, Großbritannien und Frankreich gemeinsames Handeln. Die Folge sei eine Verlagerung von Entscheidungen in ,coalitions of the willing' einzelner Staaten. Dem gegenüber seien im Sinne des ,Minilateralismus' mehrere Konstellationen der Zusammenarbeit innerhalb der GASP denkbar und anerkannt oder würden bereits praktiziert. So bestünde in der GSVP die Möglichkeit einer exklusiven vertieften Kooperation im Rahmen der Ständigen Strukturierten Zusammenarbeit (SSZ). Daneben existierten aber ebenso das Weimarer Dreieck und dessen Initiativen zur Fortentwicklung der Gemeinsamen Sicherheits- und Verteidigungspolitik (GSVP) oder die ,E3 plus 3'-Gruppe im iranischen Atomkonflikt. Unabhängig vom Erfolg dieser Modelle unterminierten sie jedoch qua Existenz den Anspruch einer gemeinsam formulierten europäischen Außen- und Sicherheitspolitik und seien damit möglicherweise als Impulsgeber zu verstehen, aber keinesfalls als das Mittel der Wahl.

Folglich hätten sich die Mitgliedstaaten nun der Gretchenfrage zu stellen, ob eine gemeinsame europäische Verteidigung überhaupt angestrebt werde oder ob diese, dem britischfranzösischen Beispiel folgend, bi- und multilateral in Kleinstgruppen organisiert werden sollte. Die letzten zehn Jahre habe ein hohes $\mathrm{Ma} 3$ an strategischer Ambivalenz den unterschiedlich engagierten Mitgliedstaaten die Akzeptanz einer gemeinsamen europäischen Politik in diesem Bereich erleichtert. Inzwischen seien differierende Ambitionen und 
wahrgenommene Defizite aber zu Problemen geworden, die den Prozess zunehmend unattraktiv wirken ließen. Sollte daran nichts substanziell geändert werden, drohe in der Folge durch die Kumulation der Unzufriedenheit eine weitergehende Erosion der gemeinsamen Politiken, wenn nationale Alleingänge den GSVP-Anspruch immer wieder infrage stellten. Wirksam entgegenzuwirken sei dem analog zur Finanz- und Wirtschaftspolitik nur durch einen ambitionierten Integrationsprozess, der eine Abkehr vom Prinzip der Freiwilligkeit bedeuten würde und operative Kosten besser auch auf Nichtteilnehmer verteilen müsse. Zusätzlich sei ein Weißbuch der gemeinsamen Verteidigung lange überfällig. Ergänzt und forciert werden könnte diese Entwicklung durch einen ,bottom-up'-Prozess der Fähigkeitsentwicklung sowie klare strategische Leitlinien innerhalb bestehender ,coalitions of the willing', die somit ihre MotorFunktion für den Rest der Union ausüben können. Einigkeit bestand abschließend darüber, dass die politische Union nicht ohne GSVP erreicht werden könne. Nichtsdestotrotz werde die Entscheidung über Krieg und Frieden auf absehbare Zeit unter dem Vorbehalt nationaler Parlamente bleiben.

Zum Abschluss des ersten Tages griff der Erste Parlamentarische Geschäftsführer der CDU/ CSU-Fraktion Peter Altmaier, $M d B$, einige der bislang diskutierten Punkte in einer Dinner Speech auf. So erörterte er den parlamentarischen Prozess um die deutsche EFSF-Ratifizierung und legte die Notwendigkeit intergouvernemental ausgehandelter Reformen dar. Dabei ging er auch auf die notwendige Überarbeitung der seit dem Vertrag von Maastricht nicht grundlegend reformierten WWU ein und schloss mit einer Einschätzung der Perspektiven für das hoch verschuldete Griechenland.

Die aktuelle deutsche Europapolitik aus Sicht der europäischen Partner

Im vierten Forum warfen die Teilnehmer einen Blick von außen auf die deutsche Europapolitik. Die Wahrnehmung externer Akteure bot interessante Diskussionsanstöße, denn die Einschätzungen der Rolle Deutschlands innerhalb der Europäischen Union rangierten von „Hegemon wider Willen“ über „kooperativer Hegemon“ bis hin zu ,de-Facto-Leader“.

Die Grundlage der Europapolitik eines jeden Staates lasse sich innerhalb eines Kontinuums von Solidarität zu Souveränität verorten. Welcher Schwerpunkt gesetzt werde, bestimme die Rolle des Staates im Integrationsprozess. Einig waren sich die Diskutanten darüber, dass sich die Rolle Deutschlands in den letzten Jahren gewandelt habe. War die Partnerschaft mit Frankreich lange Zeit Leitlinie der deutschen Europapolitik, so gab es in der jüngsten Vergangenheit mehrere Situationen, die die Unumstößlichkeit dieser kollektiven , leadership'-Struktur infrage stellten. Die Beiträge machten deutlich, dass die deutsche Politik, aus der Perspektive der Nachbarstaaten betrachtet, derzeit lediglich zwischen Alleingängen oder Enthaltung wähle.

So wurde auch in diesem Panel die deutsche Enthaltung in der Sitzung des Sicherheitsrates der Vereinten Nationen bei der Entscheidung über einen Militäreinsatz im Rahmen der UNResolution 1973 thematisiert. Dass es trotz der deutschen Zurückhaltung eine, europäische Antwort' gab, sei durch die enge britisch-französische Kooperation gewährleistet worden.

Weiterhin sei, vor allem in Frankreich, der mit den EU-Partnern nicht abgesprochene Ausstieg Deutschlands aus der Atomkraft als Alleingang gewertet worden. Dies widerspräche den Bemühungen, die Energiepolitik zu europäisieren. In Tschechien beispielsweise wäre die Sorge verbreitet, dass die Bundesregierung ihre Entscheidung, aus der Kernkraft auszusteigen, auch auf europäischer Ebene durchsetzen möchte. Auch die deutsche Politik im Zuge der Finanzkrise und die Zurückhaltung der Bundesregierung bei der Kooperation zur Rettung des Euro sorgten bei den übrigen Mitgliedstaaten für Verstimmung. Hierbei sei zwar zu berücksichtigen, dass es 
auf europäischer Ebene keine einheitliche Wirtschaftspolitik gebe, das Verhalten der Bundesregierung würde nichtsdestotrotz als nicht kooperativ empfunden. So würden in Italien die Optionen des Alleingangs und der Enthaltung als zunehmend passiv oder übermäßig vom nationalen Interesse geprägt angesehen.

Die Diskussionsteilnehmer betonten, dass das Verhalten Deutschlands in den oben genannten Fällen, welches - zumindest auf den ersten Blick - eine Abkehr von der innereuropäischen Solidarität andeutete, Teil einer gesamteuropäischen Grundstimmung sei. Die Rahmenbedingungen für die Europäische Union hätten sich in einer Weise verändert, die alte Muster der Solidarität auf die Probe stellten. Zum ersten Mal in der Geschichte stünde der Mythos vom Frieden nicht mehr als vereinendes Narrativ zur Verfügung. Die Generation der derzeitigen Akteure stünde nicht mehr unter dem Eindruck der Weltkriege, gehöre aber auch nicht zur jungen Generation derer, die Europa und alle damit verbundenen Vorteile wie die Reisefreiheit als Selbstverständlichkeit erachten. Weiterhin sei ein Trend zur Nationalisierung europäischer Politik zu verzeichnen. In einigen Bereichen sei die Union inneren Spaltungen ausgesetzt. So fordere der europäische Norden eine Stabilisierung der Währung, während Frankreich sich auf die Stabilisierung der Märkte konzentriere. Ein weiteres Beispiel für diese Spaltung könne innerhalb der Europäischen Nachbarschaftspolitik ausgemacht werden, wo die einzelnen Mitgliedstaaten den Regionen eine unterschiedliche Bedeutung zumessen.

Die Erwartung einer Führungsrolle werde von außen an Deutschland herangetragen, während die Handlungsspielräume zunehmend kleiner ausfielen. So sei die deutsche Politik durch eine Vermeidung von ,leadership“ gekennzeichnet oder werde, wie im Zuge der Finanzkrise, als Alleingang oder Egoismus interpretiert. Die Rolle Deutschlands als Garant für Stabilität hingegen werde bisweilen überschätzt. Die Diskussionsteilnehmer stellten heraus, dass es nicht ausschließlich die Ausgestaltung der Politik selbst sei, die zu Charakterzuschreibungen führe, sondern der Diskurs, der darüber im Inland, aber vor allem im Ausland geführt werde.

Die Frage, welches ,Etikett ‘ die gegenwärtige Politik Deutschlands am besten beschreibt, konnte nicht abschließend oder einstimmig beantwortet werden. Klar wurde jedoch, dass Deutschlands Partner innerhalb der Europäischen Union einen Wandel der deutschen Politik wahrnehmen.

Die Rolle von Bundestag und Verfassungsgericht bei den Beschlüssen zur Bekämpfung der Eurokrise

Das letzte Panel untersuchte das Wirken von Bundestag und Bundesverfassungsgericht im Zuge der Eurokrise und diskutierte die sich daraus ergebenden möglichen ,Vetospieler'Rollen dieser Institutionen. Besonderes Augenmerk wurde auch auf die Frage gelegt, in welchem Ausmaß nationale Parlamente und Gerichte angesichts so weitreichender Entscheidungen in diesen intergouvernementalen Prozess konstruktiv eingebunden werden könnten und sollten.

Einigkeit bestand seitens der Diskussionsteilnehmer zunächst darüber, dass die Rolle des Bundesverfassungsgerichts durch eine Charakterisierung als ,Vetospieler' unzulässig verkürzt würde. Während der Mitwirkungsgrad durch die Politikfelder hindurch differiere und etwa in der Energiepolitik anders gelagert sei als in der Finanz- und Wirtschaftspolitik, belasse es das Verfassungsgericht nicht dabei, Grenzen zu ziehen. Durch seine Entscheidungen und Interpretationen gestalte es vielmehr Politik mit und übersteige somit die Rolle des klassischen, Vetospielers'. Insbesondere die Eurokrise verdeutliche dabei zwei parallel zu beobachtende Tendenzen, nämlich eine zunehmende Verrechtlichung der Politik und eine Politisierung der Rechtsprechung. So warte der Bundestag beispielsweise mitunter bei Gesetzentwürfen auf 
das Urteil des Bundesverfassungsgerichts und verzichte somit auf den ihm zugedachten Gestaltungsspielraum. Gleichzeitig habe das Gericht dem Parlament in manchen Fällen wortwörtlich Gesetzestexte diktiert und damit seinen Anspruch als Mitgestalter deutscher Europapolitik untermauert.

Auch der Deutsche Bundestag wurde, unter anderem mit Verweis auf Art. 12 des Vertrags über die Europäische Union in der Fassung des Vertrags von Lissabon, nicht als ,Vetospieler' gesehen. Da es einen Unterschied zwischen politischen und rechtlichen Entscheidungen gebe, sei die Gestaltungsmacht von Parlamentariern und Richtern trotz gewisser Näherungstendenzen sehr unterschiedlich. So hätte das Europa-Referat der Verwaltung des Bundestages in den letzten 15 Monaten etwa 30.000 Dokumente mit Bezug zur Europäischen Union zu bearbeiten gehabt, wozu noch das Zehnfache an Folgedokumenten sowie 870 parlamentarische Anfragen kämen. Dies zeige die Dimension der kaum bewältigbaren Informationsflut, mit der die Abgeordneten konfrontiert seien. Infolge dessen sei die Aufgabe des Referats auf die reine Bearbeitung und Weiterleitung ausgewählter Dokumente reduziert, von einer, Vetospieler'-Rolle der Verwaltung könne allein daher schon nicht die Rede sein. Die hochkomplexe Materie sei eine inhaltliche und logistische Herausforderung für das Gremium. Zeitgleich müsse der Bundestag aber auch mit der neuen Phase der Integration Schritt halten und seine Stellung im politischen System behaupten. Dies gelte allerdings für alle nationalen Parlamente, welche zukunftsträchtige Antworten auf die intergouvernementale Weiterentwicklung Europas finden und gleichzeitig ihre verfassungsgemäßen Aufgaben als Repräsentanten der Bevölkerung bewahren müssten.

Der gestiegene Einfluss des Bundesverfassungsgerichts wurde im Kontext der Diskussion über die abnehmende Gestaltungsmacht der Bundestagsabgeordneten in der Europapolitik abschließend mit Sorge betrachtet. Während es etwa das Konzept der nationalen Souveränität weitaus rigider auslege und engere Spielräume für die europäische Integration definiere als es vom Grundgesetz intendiert sei, sollte der Bundestag als Vertreter des Souveräns selbstbewusster agieren und die ihm zugedachte Rolle im Gesetzgebungsprozess freier ausüben können. Dazu müsse der Bundestag seine Rechte stärker wahrnehmen, anstatt sich getreu dem Motto ,Abwarten, was Karlsruhe dazu sagt ' beständig am Bundesverfassungsgericht zu orientieren. Idealerweise sollte demzufolge zwischen Parlament und Verfassungsgericht kein Machtkampf, sondern eine symbiotische Beziehung zweier starker staatstragender Organe bestehen, in der der Bundestag dem Anspruch demokratischer Legitimation Genüge tue, während das Bundesverfassungsgericht den europäischen Integrationsprozess im Sinne des Grundgesetzes konstruktiv begleite.

Zusammenfassend lässt sich feststellen, dass die gegenwärtige Häufung politischer und wirtschaftlicher Umbrüche für alle Akteure nicht nur in Deutschland, sondern auch in der gesamten Europäischen Union - Neuland bedeutet. Dabei werden die zugrunde liegenden Konflikte in Deutschland als dem größten Geldgeber innerhalb der Europäischen Union und wichtigem außenpolitischen Akteur besonders deutlich. Die Nachbarstaaten nahmen mit Blick auf das deutsche Krisenmanagement dabei eine gewisse Abkehr vom bisherigen, verlässlich pro-europäischen Kurs wahr: Deutschlands gegenwärtiges Handeln sei stärker durch wirtschaftliche Eigeninteressen und mangelnde Abstimmung mit den Partnern gekennzeichnet. Dabei stehe Deutschland auch innerhalb der Europäischen Union nicht alleine da, sondern sei oftmals Repräsentant eines neuen Selbstbewusstseins der wirtschaftlich stärksten Staaten. 